\title{
Dietary intervention with an Okinawan-based Nordic diet in type 2 diabetes rendered decreased interleukin-18 concentrations and increased neurofilament light concentrations in plasma
}

\author{
Clara Nilholm¹, Bodil Roth ${ }^{1}$, Peter Höglund ${ }^{2}$, Kaj Blennow, Elisabet Englund, Oskar \\ Hansson, Henrik Zetterberg, Bodil Ohlsson ${ }^{1}$
}

${ }^{1}$ Lund University, Skane University Hospital, Department of Internal Medicine, Malmö,

Sweden

${ }^{2}$ Department of Clinical Chemistry \& Pharmacology, Skane University Hospital, Lund University,

Lund, Sweden

\author{
Kajs adresser: \\ ${ }^{1}$ Department of Psychiatry and Neurochemistry, Institute of Neuroscience and \\ Physiology, the Sahlgrenska Academy at the University of Gothenburg, \\ Mölndal, Sweden \\ ${ }^{2}$ Clinical Neurochemistry Laboratory, Sahlgrenska University Hospital, \\ Mölndal, Sweden
}

Henriks addresser:

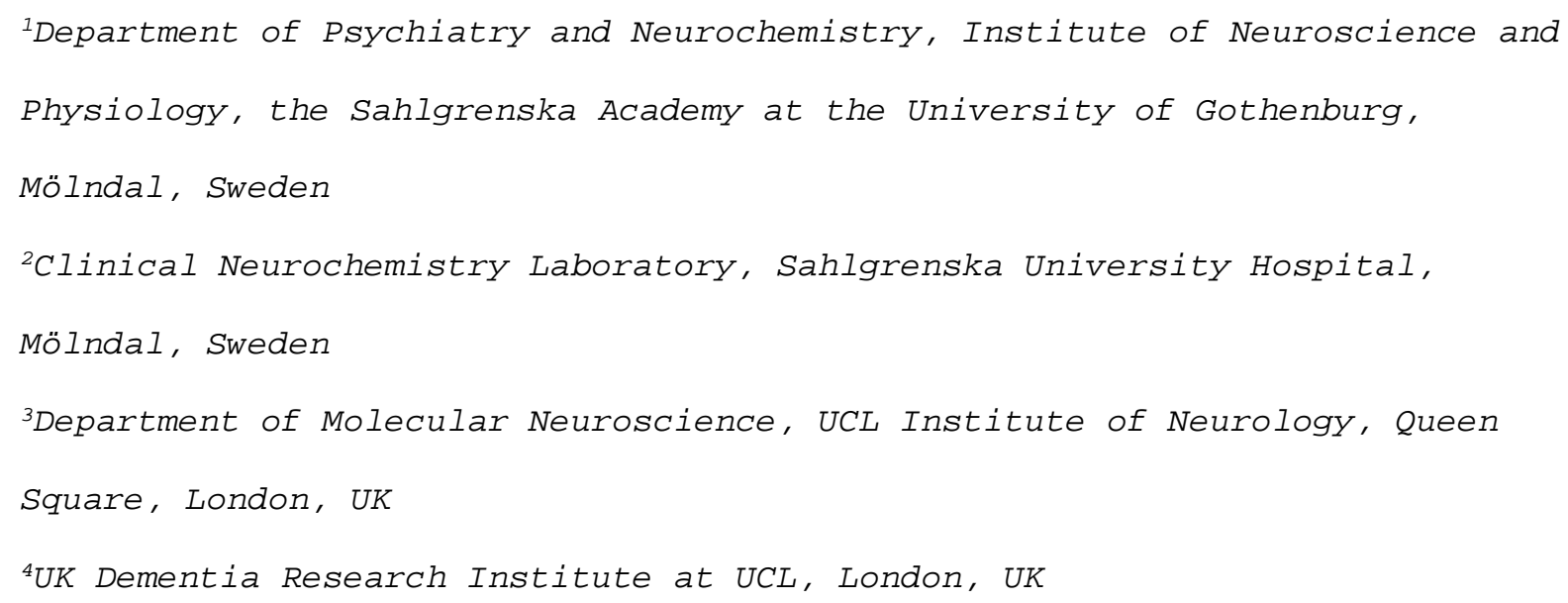




\title{
Corresponding author
}

\author{
Bodil Ohlsson \\ Lund University \\ Skåne University Hospital \\ Department of Internal Medicine \\ Jan Waldenström Street 15 \\ S-205 02 Malmö, Sweden \\ Phone: +4640331000 (switchboard) \\ Fax: +4640336208 \\ E-mail: bodil.ohlsson@med.lu.se
}

\begin{abstract}
Diabetes mellitus is characterized by low-grade inflammation and cognitive impairment. An Okinawan-based Nordic (O-BN) diet rendered improved anthropometry and metabolism. The aim of this study was to examine the inflammatory and neural responses after O-BN diet in health and type 2 diabetes (T2D). Two different breakfasts; one standard and one O-BNbased, were given in random order to 19 healthy volunteers. A 12-week O-BN-dietary intervention in T2D was performed, and the participants were followed for another 16 weeks, with registration of anthropometry and metabolic parameters. Non-diabetic subjects served as controls at baseline. Plasma was analyzed for cytokines by a 10-plex Luminex assay and for neurofilament light (NfL) by an ultrasensitive Single molecule array (Simoa) assay. Cytokine levels decreased after a single breakfast intake, independent of composition. Cytokine levels
\end{abstract}


were higher in T2D than in controls. Anthropometric and metabolic parameters were improved by the dietary intervention. In parallel, cytokine levels were lowered, although only significantly for IL-18 $(\mathrm{p}=0.001)$ and with a tendency for IL-12p70 $(\mathrm{p}=0.07)$. IL-18 levels correlated with fasting glucose, HbA1c and lipids, but did not correlate with body mass index, insulin levels or blood pressure. NfL levels increased during the intervention ( $p=0.049)$. In conclusion, an O-BN-dietary 12-week intervention in T2D rendered decreased circulating IL18 levels, which correlated with improved metabolic markers, but no beneficial effect on a blood-based neurodegeneration marker in the form of NfL.

Keywords: cytokines, IL-18, inflammation, neurofilament, Okinawan-based Nordic diet, type 2 diabetes

\section{Introduction}

Obesity, the metabolic syndrome and metabolic disease such as type-2 diabetes (T2D) have previously been shown to be associated with low-grade chronic inflammation in both animal and human trials [1]. Whether chronic low-grade inflammation is a precursor to metabolic disease remains undetermined, and longitudinal interventional studies are needed in order to explore causality. Diet has been proposed to affect expression of inflammatory parameters; with some nutrients believed to act pro-inflammatory and others anti-inflammatory [2]. The typical Western diet with a high carbohydrate intake has been thought to generate low-grade chronic inflammation [2], while a healthier diet with a high proportion of fiber, e.g. the Okinawanbased Nordic (O-BN) diet, would hypothetically decrease systemic inflammation [3] . We have 
previously shown that a single meal based upon the O-BN diet induced lower postprandial glucose, insulin and triglyceride levels compared to a standard meal in healthy volunteers [4, 5]. In yet another publication, we reported elevated calprotectin and zonulin serum levels in T2D patients after a 12-week dietary intervention with the O-BN diet, whereas haptoglobin and thrombocyte levels were lowered [6]. Since study participants experienced improved anthropometry and metabolism [7], these results were somewhat surprising. It is, however, not necessarily certain that the observed elevations in calprotectin and zonulin stem from the change in dietary composition. Another possibility is that these results were due to liver impact secondary to weight loss. Furthermore, no consensus has been reached as to which biomarkers best reflect, and should be used in determining, presence of chronic low-grade inflammation [8]. Bearing this in mind, it seems important to investigate dietary intervention effect on a multitude of inflammatory parameters.

In the T2D population, cognitive decline is more commonly seen than in healthy populations [9]. There are several proposed pathologic mechanisms linking T2D to neural damage, such as chronic hyperglycemia and glucose toxicity resulting in osmotic insults and oxidative effects on neurons, ischemia of the microvascular system, and insulin metabolic dysfunction promoting amyloid- $\beta$ accumulation in the brain [9-11]. Indeed, similarities have been observed between Alzheimer's disease (AD) and T2D neuropathology, including reduced insulin signaling and insulin-like growth factor abnormalities in the AD brain [12]. T2D is a significant risk factor for developing cognitive impairment as well as $\mathrm{AD}$ [10). Diabetes duration, glycated hemoglobin (HbA1c) levels and glycemic fluctuations have been shown to be associated with cognitive decline and dementia [9]. Neurofilament light (NfL) is a structural protein that is highly expressed in large caliber myelinated axons that extend subcortically (ref: PMID: 27387643). Neurodegeneration results in release of the protein into the brain interstitial fluid that communicates freely with the cerebrospinal fluid (CSF). 
Increased CSF NfL concentrations are seen in a broad range of neurodegenerative diseases, including AD (ref: PMID: 27387643). Recent advances in ultrasensitive measurement technologies have made it possible to measure NfL in blood. Using a Single molecule array (Simoa)-based method, NfL can be measured in blood with robust correlation with CSF (ref: PMID: 26870824). AD patients have increased blood NfL concentrations and the levels correlate with the onset and intensity of the neurodegenerative process [13]+ PMID: 29070659. We wanted to examine if the O-BN dietary intervention, which led to improved fasting glucose, HbA1c and insulin levels, along with improved health-related quality of life (HRQoL) with greater physical functioning and vitality [7], had any effect on this potential blood-based biomarker.

To further investigate the effect of dietary intervention on inflammatory and neural response, two separate dietary interventional studies with the O-BN diet were performed, where an assay of cytokine and NfL levels were analyzed in plasma. The study population, design and diet of both studies have been thoroughly described in previous publications $[4,5$, 7]. The aim of the present part-study was to examine response of circulating cytokines to dietary intervention with the O-BN diet both postprandially in healthy individuals and after a 12-week dietary intervention in the specific study population of T2D patients. Additionally, we wanted to examine levels of the neural blood-based biomarker NfL in T2D patients before and after dietary intervention with the O-BN diet.

\section{Material \& Methods}

The subjects were treated according to the Helsinki declaration and the study was approved by the Regional Ethics Review Board at Lund University (2014/460). All subjects gave their written, informed consent before entering the study, which was monitored by an external monitor and registered at ClinicalTrials.gov data base (NCT02405806). 


\section{Study population}

\section{Breakfast study}

In the breakfast study examining the effect of a single O-BN diet meal intervention, 20 healthy volunteers (8 men and 12 women; mean age (in years) \pm standard deviation (SD): $46.0 \pm 14.5$; mean body mass index $\left[\mathrm{BMI}\right.$; in $\left.\left.\mathrm{kg} / \mathrm{m}^{2}\right] \pm \mathrm{SD}: 24.6 \pm 2.7\right)$ with no symptoms or history of severe gastrointestinal disease and no history of abdominal surgery or diabetes mellitus were included. Study participants were recruited from staff and staff family members. Additional information on inclusion and exclusion criteria are presented in previous publications by our study group $[4,5]$.

\section{Dietary interventional study}

In total, 45 patients diagnosed with T2D were randomly selected from a health care center in the south of Sweden for the 12-week O-BN dietary interventional study, after consideration of inclusion and exclusion criteria of other concomitant severe diseases. Finally, 30 patients (13 men and 17 women; mean age [in years] \pm SD: $57.5 \pm 8.2$; mean BMI [in kg/m²] \pm SD: $29.9 \pm$ 4.1) were recruited. Reasons for not inclusion were: unwillingness to participate $(n=11)$, late autoimmune diabetes in adult (LADA) $(\mathrm{n}=1)$, a history of gastric by-pass surgery $(\mathrm{n}=1)$, pregnancy $(n=1)$, or already on a diet $(n=1)$. The disease duration of type 2 diabetes was $10.4 \pm 7.6$ years. The treatment was metformin $(40 \%)$, metformin in combination with insulin $(27 \%)$, insulin solely (13\%), metformin in combination with sulfonylurea (7\%), diet solely (7\%), sulfonylurea (3\%), or dipeptidyl peptidase-4 (DPP) (3\%). The most common secondary complication to the disease was autonomic neuropathy and/or peripheral neuropathy (30\%), retinopathy (27\%), and nephropathy and macroangiopathy (17\% in both). Only one patient had a verified gastroparesis. Out of the 30 patients originally included in the study, all remained at week 12 and 23 patients remained at week 28. Additional information on 
inclusion and exclusion criteria are presented in previous publications by our study group [6, 7].

Non-diabetic controls without gastrointestinal disease (25 women; mean age [in years $] \pm \mathrm{SD}, 64.7 \pm 5.3$; mean BMI $\left[\right.$ in $\left.\left.\mathrm{kg} / \mathrm{m}^{2}\right] \pm \mathrm{SD}: 24.1 \pm 3.6\right)$ were gathered from previously collected study data for comparison at baseline.

\section{Study design}

\section{Breakfast study}

The trial was designed as a crossover study (Figure 1). All tests were performed under standardized conditions and stable temperatures. Study participants were examined between 7:45 and 11:15 AM after a 10-h fast. Alcohol intake and intensive physical activity were not permitted the day before each breakfast, and participants were informed to eat meals as similar as possible on the day prior. All participants received two different breakfasts on 2 different days (Table 1). The two meals averaged the same caloric intake but differed in food item and nutrient composition (further described below). They were served in a random order at 1-week intervals and were to be ingested within 15 minutes. For further information on the study design, see previous publications $[4,5]$.

\section{Dietary interventional study}

The trial was designed as a longitudinal prospective study (Table 1). T2D patients received the O-BN diet; a diet inspired by the Okinawan diet, but based on traditional Nordic raw food, (further described below) for 12 weeks, followed by an additional 16-week observational period during which participants chose their own diet. Participants were instructed to maintain their regular physical activity habits throughout the intervention. Questionnaires about sociodemographic factors, lifestyle habits and medical history; anthropometric variables and blood samples were collected at baseline, 12 and 28 weeks. For further information on the study design, see previous publications $[6,7]$. 


\section{Test meals}

\section{Breakfast study}

The reference breakfast was composed of low-fat yogurt with strawberry flavor and granola, 1 slice of rye bread $(6.5 \mathrm{~g}$ fiber/100 $\mathrm{g})$, butter spread containing $60 \%$ fat, hard cheese containing $28 \%$ fat, 1 glass of orange juice, and 1 cup of either coffee or tea, amounting to a glycemic load of 34. The O-BN test breakfast consisted of filmjölk (a Swedish yoghurt-like fermented product) natural flavor (fat content: $1 \%$ ), with frozen strawberries and muesli made at home, 1 slice of whole grain rye bread with sun flower seeds $(9.1 \mathrm{~g}$ fiber/100 $\mathrm{g})$, butter spread containing $60 \%$ fat, hard cheese containing 17\% fat (20 g), smoked turkey, vegetables, 1 boiled egg, 1 cup of either coffee or tea, and 1 glass of tap water, amounting to a glycemic load of 14 . The participants began the meal by chewing the vegetables carefully before the remainder of the meal was subsequently eaten. The test meal had a $38 \%$ lower carbohydrate content, $44 \%$ higher fat content (mono-unsaturated fat: $5.8 \mathrm{~g}$ vs. $2.3 \mathrm{~g}$; poly-unsaturated fat: $4.4 \mathrm{~g}$ vs $0.3 \mathrm{~g}$ ), and $53 \%$ higher protein content than the reference meal. The reference breakfast constitutes a standard Swedish breakfast and was based on the traditional recommendations by the Swedish National Food Agency for patients with diabetes, whereas the O-BN test breakfast resembled a moderately low-carbohydrate diet, which is an alternative recommendation [14].

\section{Dietary interventional study}

Two meals a day, and snacks consisting of a variety of fruits, berries, and seeds, were delivered to the subjects throughout the study free of charge, along with recipes and instructions on how to prepare the food. The participants only had to purchase their own breakfast, which consisted of either porridge or fermented milk depending on the participant's regular breakfast. The O-BN diet was based on the traditional Okinawan diet, but modified with tastes and food components to suit the Nordic population [7]. The meal composition was close to a moderately low carbohydrate-rich diet with higher contents of fiber, fat and protein; which constitutes the above-mentioned alternatively recommended diet by the Swedish 
National Food Agency for patients with diabetes [14]. These recommendations are also in line with international recommendations $[15,16]$. The food is based on traditional Nordic raw foods such as whole grains, vegetables, legumes, and root crops as well as fat fish, poultry, fruits, berries, and nuts. Food items were minimally industrially processed and organic when possible. In addition, the amount of sugar, white flour, red meat, processed meat, and dairy products was limited. The content of high glycemic index foods was reduced (e.g. white flour and sugar) in favor of nutrients with low impact on blood sugar. The diet had a good nutritional supply with a mean calorie intake of about $1,900 \mathrm{kcal} / \mathrm{day}$. The food composition consisted of 42 Energy percentage (E\%) carbohydrates with 4 E\% of dietary fibers, $23 \mathrm{E} \%$ protein, $35 \mathrm{E} \%$ fat, with $10 \mathrm{E} \%$ of saturated fatty acids, $10 \mathrm{E} \%$ of mono-unsaturated fatty acids and $8 \mathrm{E} \%$ of poly-unsaturated fatty acids. The levels of vitamins and minerals were higher than the lowest levels recommended [7, 14]. No other changes in diet or supplements were allowed to be introduced during the study period. Maximal intake of alcoholic beverages was set to $30 \mathrm{~g}$ ethanol/week.

Most study participants coped well with the new diet. In some individual cases, however, adjustments to the diet had to be made for different reasons. These included a decrease in fiber content in four study participants with irritable bowel syndrome (IBS) who suffered from gastrointestinal symptoms, and an increased caloric content in five participants who experienced rapid weight loss.

\section{Blood samples}

Venous blood samples were collected through an intravenous catheter 10 minutes before the breakfast meals, immediately before the meals, and 10, 20, 30, 45, 60, 90, 120, and 180 minutes after the meals. In the dietary interventional study, samples were taken in the morning between 07:45 and 09:00 h after a 10-h fast. All samples consisted of whole blood drained into 6-mL glass tubes, serum separation tubes with coagulation activator and gel or plasma separation 
tubes with ethylenediaminetetraacetic acid (EDTA) (BD Microtainer, Franklin Lakes, NJ, USA). Blood samples were immediately cooled and centrifuged at $3000 \mathrm{rcf}$ for 5 minutes. The plasma was harvested and stored at $-80^{\circ} \mathrm{C}$ until analyzed for cytokine and NfL concentrations. Samples were not thawed between initial storage and analysis. Hemoglobin, leukocytes, thrombocytes and hemoglobin A1c (HbA1c) in blood; albumin, C-reactive protein (CRP), glucose, cholesterol and triglycerides in plasma; and insulin in serum were analyzed by standard methods at the Department of Clinical Chemistry at Skåne University Hospital, Malmö, Sweden.

\section{Plasma analyses of cytokines}

The concentrations of TNF- $\alpha$, IL-1 $\beta$, IFN- $\gamma$, IL-2, IL-12 p70, IL-18, IL-4, IL-1 $\alpha$, IL-10 and IL-17A were quantified in un-diluted plasma using a custom premixed human magnetic 10plex. Luminex assay (R\&D Systems, Minneapolis, MN, USA, No 1434853 and 1447942). The Luminex assay was performed according to manufacturer's instructions. Briefly, samples were pipetted into appropriate wells in the Luminex plate and incubated with microparticle cocktail for 2 hours at room temperature. Each well was washed three times using a magnetic device (Bio-Plex Handled magnetic washer, 171020100, Bio-Rad Laboratories, Hercules, CA, USA). Biotinylated detection antibodies were added to form a sandwich complex, followed by another three washes. The final detection complex was created with the addition of streptavidinphycoerythrin (SA-PE) conjugate. After a final wash and resuspension, the plate was analyzed using a Bio-Plex 200 system reading 50 events/bead at a flow rate of $60 \mu 1 /$ minute with doublet discriminator gates at 8,000 and 16,500 (Bio-Rad Laboratories). Absolute concentrations $(\mathrm{pg} / \mathrm{mL})$ were measured from standards provided with the kit. All samples were analyzed in duplicate and the concentration of cytokine bound to each bead was proportional to the median fluorescence intensity (MFI) of reporter signal. Standard curves were calculated with nonlinear 
regression type 5-parameter logistic. Inter-assay and intra-assay coefficients of variation (cv) were $3.47 \%$ and $5.40 \%$, respectively.

\section{Plasma analyses of $\mathrm{NfL}$}

Plasma NfL concentration was measured using a previously described in house Simoa assay (ref: PMID: 27581216). Intra- and inter-assay coefficients of variation were 8.2-8.7\%.

\section{Statistical analyses}

IBM SPSS Statistics 24 was used for analysis of the collected data. Inflammatory parameters and NfL were found to not have a normal distribution after application of the KolmogorovSmirnov test. A p-value $<0.05$ was considered statistically significant.

A power analysis was performed to establish the number of participants required to demonstrate clinically important differences in metabolic parameters, systolic and diastolic blood pressure and weight. It was determined that 9 participants were required for the breakfast study, and 18 subjects were required for the dietary interventional study, in order to demonstrate clinically significant differences with $80 \%$ power at $5 \%$ significance level.

For one participant in the breakfast study, several samples for the Luminex analyses in the reference experiment were missing, leaving 19 participants in the analyses. Cytokines IL-1 $\alpha$, IL-10, and IL-17A were excluded due to many non-detectable concentrations. Missing concentrations from cytokines included in analyses were replaced with a value of 0.01 . In the dietary interventional study, cytokines IL-10 and IL-17A were excluded. For baseline comparison between diabetic and non-diabetic controls, cytokines IL$1 \alpha$, IL-1 $\beta$, IL-10 and IL-17A were excluded. Non-detectable concentrations of the remaining cytokines were replaced with randomized numbers between zero and the lowest value measured for each individual cytokine. Excel 2016 was used for number randomization. 
In the breakfast study, we tested the hypothesis that two different breakfasts would result in different cytokine responses in the same group of study participants by using the non-parametric Wilcoxon test. Baseline values, maximum and minimum concentrations and delta-values for maximal and minimal changes from baseline were determined and presented as median values and median of differences along with interquartile ranges.

In the dietary interventional study, we tested the hypothesis that dietary intervention would result in altered levels of cytokines and NfL in study participants with linear mixed effect models. Continuous variables were analyzed, with random intercept and unstructured covariances for repeated measures within a patient, with visits as nominal fixed effect, using baseline as reference. Mean values and their $95 \%$ confidence limits are presented, together with estimates of changes from baseline and 95\% confidence limits. Baseline comparisons between T2D patients and healthy, non-diabetic individuals were calculated using the Student t-test or Mann-Whitney U-test.

Anthropometric and metabolic parameters are presented as mean and standard deviation (SD) according to previous publications, and calculated by paired t-test in the breakfast study and by linear mixed effect models in the interventional study [4, 5] . Correlations between anthropometric and metabolic parameters and cytokine levels were performed by Spearman's correlation test.

\section{Results}

\section{Breakfast study}

Baseline levels of plasma glucose did not differ between the two study days (5.40 \pm .58 and $5.28 \pm 0.42 \mathrm{mmol} / \mathrm{L}$, respectively). A lower postprandial maximal plasma glucose concentration was observed after the test breakfast $(6.49 \pm 0.77 \mathrm{mmol} / \mathrm{L}$ vs. $7.37 \pm 0.84$ $\mathrm{mmol} / \mathrm{L}, \mathrm{p}<0.001)$, and a lower change from baseline $(1.09 \pm 0.54 \mathrm{mmol} / \mathrm{L}$ vs. $2.09 \pm 0.79$ 
$\mathrm{mmol} / \mathrm{L}, \mathrm{p}<0.001$ ), compared with the reference breakfast. Baseline levels of triglycerides were equal between the two days $(1.01 \pm 0.28$ and $1.02 \pm 0.30 \mathrm{mmol} / \mathrm{L}$, respectively). The maximal postprandial triglyceride levels did not differ between test and reference breakfast $(1.28 \pm 0.42$ vs. $1.25 \pm 0.40 \mathrm{mmol} / \mathrm{L}, \mathrm{p}=0.669)$. However, the delta area under the curve (dAUC) was less markedly increased after the low-carbohydrate test breakfast $(13.8 \pm 21.1$ $\mathrm{mmol} / \mathrm{L}, \mathrm{p}=0.011)$ than after the control breakfast $(19.7 \pm 18.6 \mathrm{mmol} / \mathrm{L}, \mathrm{p}<0.001)$, but the dAUCs did not differ between the groups $(\mathrm{p}=0.277)$.

\section{Dietary interventional study}

During the 12-week study period, mean BMI decreased from $29.9 \pm 4.1 \mathrm{~kg} / \mathrm{m}^{2}$ to $27.8 \pm 3.9$ $\mathrm{kg} / \mathrm{m}^{2}(\mathrm{p}<0.001)$, and this change was mainly retained at week 28 . Fasting plasma glucose levels decreased from $9.7 \pm 3.5 \mathrm{mmol} / \mathrm{L}$ to $7.9 \pm 2.7 \mathrm{mmol} / \mathrm{L}$ after 12 weeks $(\mathrm{p}<0.001)$, but increased again after 28 weeks. HbA1c levels decreased from $61.6 \pm 17.6 \mathrm{mmol} / \mathrm{mol}$ to $49.2 \pm$ $10.4 \mathrm{mmol} / \mathrm{mol}(\mathrm{p}<0.001)$ after 12 weeks and to $54.4 \pm 13.3 \mathrm{mmol} / \mathrm{mol}$ after 28 weeks $(\mathrm{p}=$ 0.002). Serum insulin levels were decreased after 12 weeks $(\mathrm{p}<0.001)$ and increased again after 28 weeks. Both cholesterol and triglyceride plasma levels were lower after 12 weeks (4.7 \pm 0.8 vs. $4.2 \pm 1.0 \mathrm{mmol} / \mathrm{L}, \mathrm{p}<0.001$ and $1.8 \pm 1.1$ vs. $1.5 \pm 0.9 \mathrm{mmol} / \mathrm{L}, \mathrm{p}=0.009$ respectively), but returned to basal values at week 28 (Table 2).

During the interventional period, one subject had hernia surgery, and another went through hip arthroplasty. Some subjects suffered airway $(n=6)$, teeth $(n=1)$ and urinary tract $(n=1)$ infections, which did not affect the concentrations of any inflammatory biomarkers (data not shown). During the follow-up, airway $(n=1)$ and teeth $(n=1)$ infections were prevalent. One subject experienced severe exacerbation of psoriasis throughout the study. Two subjects who were under treatment for severe depression interrupted participation at week 12. Almost all patients had kept their regular meal order with the same intake of 
breakfast as during the intervention at week 28 , and with an increased intake of vegetables and legumes. The composition of lunch and dinner was partly withheld.

\section{Inflammatory parameters}

\section{Breakfast study}

The number of non-detectable cytokine concentrations at baseline or peak concentration (maximum value) ranged from 0 (IL-18, baseline) to 6 (IL-1 $\beta$, baseline and maximum concentrations) and was, on average, 4.2 for a specific time-point (baseline or maximum values; data not shown). Missing concentrations at different time points were, for the most part, from the same individual.

An increase in concentration from baseline to maximal level was significant for IFN- $\gamma$ which increased from $667(1.2-1230)$ to $957(59.2-1370) \mathrm{pg} / \mathrm{mL}(\mathrm{p}=0.013)$ and borderline significant for TNF- $\alpha$ which increased from $29.8(0.65-53.4)$ to $30.5(2.3-63.7) \mathrm{pg} / \mathrm{mL}$ (p $=0.053)$ in the test breakfast group. A decrease in concentration from baseline to minimum level was significant for all the cytokines (except IL-2 in the reference group) in both the test and reference group (Table 3). Minimum levels were most frequently measured at 60 or 120 minutes after breakfast intake (true for all cytokines except IFN- $\gamma$ in the test breakfast group; data not shown). No significant differences in baseline, maximum, minimum, delta-max or delta-min levels of cytokines could be observed between the two groups (Table 3).

\section{Dietary interventional study}

The number of non-detectable concentrations at all time-points (baseline, 12 weeks and 28 weeks; 83 possible observations) ranged from 0 (IL-18) to 45 (IL-2) and was, on average, 18.3 per cytokine (data not shown). Most of the missing concentrations at different time points were from the same individual, albeit not exclusively.

Cytokine concentrations differed significantly between T2D patients and nondiabetic controls for all cytokines except IL-18 at baseline (Table 4). Median concentrations of 
TNF- $\alpha$, IFN $\gamma$, IL-12p70, IL-18 and IL-4 were higher in the T2D patient group $(p<0.001, p=$ $0.018, \mathrm{p}<0.001, \mathrm{p}=0.30$ and $\mathrm{p}<0.001$ respectively) (Table 4) while the median concentration of IL-2 was lower ( $\mathrm{p}<0.001)$ (Table 4) compared to the non-diabetic control group. Out of the other inflammatory parameters analyzed; including hemoglobin, leukocytes, thrombocytes, albumin, and C-reactive protein, only hemoglobin concentrations differed significantly between T2D patients and non-diabetic controls at baseline (Table 4).

The overall trend was a decrease in the concentrations of all cytokines except IL2 after the 12-week dietary intervention (Table 5). This was statistically significant for IL-18 which decreased from $226(173-280)$ to $189((136-242) \mathrm{pg} / \mathrm{mL}(\mathrm{p}=0.001)$ (Table 5) and borderline-significant for IL-12p70, which decreased from 490 (223-756) to 415 (148-682) $\mathrm{pg} / \mathrm{mL}(\mathrm{p}=0.07)$ (Table 5). Between week 12 and the follow-up at week 28, a trend towards an increase in all cytokines was observed. This increase was not statistically significant for any of the cytokines (Table 5).

The decrease of IL-18 was parallel with the decrease of glucose, HbA1c and lipid levels, whereas BMI, insulin levels and blood pressure did not correlate significantly with IL18 levels at any time point (Table 6).

\section{NfL analyses}

NfL concentrations differed non-significantly between T2D patients and non-diabetic controls at baseline (16.1 (10.6-26.4) pg/mL vs. $21.0(16.8-32.4) \mathrm{g} / \mathrm{mL}, \mathrm{p}=0.075)$ (Table 4). A significant increase in NfL concentration, from $21.0(15.3-26.7)$ to $26.2(18.5-33.8) \mathrm{pg} / \mathrm{mL}$, was seen after the 12-week dietary intervention $(\mathrm{p}=0.049)$ (Table 4). Between 12 and 28 weeks there was a non-significant decrease to 23.4 (16.7-30.2) $\mathrm{pg} / \mathrm{mL}$ (Table 5).

\section{Discussion}


In the present study, cytokine concentrations decreased significantly after both breakfast meals in healthy volunteers, with no difference in postprandial inflammatory status between the two meals. Cytokine concentrations, except IL-2, decreased after the O-BN dietary intervention in $\mathrm{T} 2 \mathrm{D}$, and this change was statistically significant for IL-18 and border-line significant for IL12p70. The decrease of IL-18 levels was parallel with the decrease of fasting glucose, HbA1c and lipid levels. NfL concentrations increased significantly during the dietary intervention.

Obesity, insulin resistance and T2D appear to be associated with abnormal cytokine production as well as upregulated acute-phase reactants and other mediators, thus constituting states with underlying chronic low-grade inflammation [1]. A frequently described pathogenetic model for metabolic disease relates to adipose tissue, and its role as an active endocrine organ affecting inflammation through macrophage-derived substances, hormonal activity and enhanced production of pro-inflammatory cytokines, such as TNF- $\alpha$ and IL-6 [17].

When comparing baseline cytokine concentrations between T2DM patients and healthy volunteers at baseline, the former group showed significantly higher levels of TNF- $\alpha$, IFN- $\gamma$, IL-12p70 and IL-18 than the latter. Although the functions of, and interactions between, these cytokines are complex, they are considered to be predominantly proinflammatory (18]. However, IL-2, which was lower in the T2D group, is described in the literature as having an immunoregulatory function. IL-2 thus helps maintain homeostasis of the immune system, and prevents autoimmunity by its effect on regulatory T-cells [19, 20]. Hence, the differences in cytokine expression between T2D patients and healthy individuals observed in this study support earlier evidence of a chronic inflammatory state in metabolic disease.

$\mathrm{IL}-18$ is considered to be the best pro-inflammatory biomarker in metabolic diseases, compared to other cytokines [21]. The dietary intervention resulted in improved 
anthropometric and metabolic parameters [7], which in the present study were correlated with reduced IL-18 levels. Similar results have been found in other dietary interventional studies including a 2-year dietary intervention with a Mediterranean style diet (60 obese women) [22], a 15-week lifestyle intervention (hypocaloric diet and daily exercise;

27 severely obese men) [23] and a 3-year dietary counselling and n-3 fatty acid supplementation study (563 elderly high-risk men) [24], and a long-term study with healthy Nordic diet (213 patiets with metabolic syndrome) [25]. Bruun et al. [23] found that serum levels, but not adipose tissue concentrations, of IL-18 decreased; leading the writers to conclude that improved insulin sensitivity, and not weight loss, was responsible for the changes observed. Fasting glucose levels were independently associated with adipose tissue concentrations of IL-18 mRNA in another study [26]. Furthermore, large prospective studies have provided evidence of an association between IL-18 and increased risk of future T2D development, independent of risk factors such as BMI and adipokine levels [27, 28]. The decrement of IL-18 during the present dietary intervention, which coincided with improved glucose and lipid metabolism, is interesting and provides further evidence of the associations between diet, inflammation and metabolic disease. We have previously published that hemoglobin, leukocytes, CRP and albumin were unaffected throughout the O-BN interventional study, but lower levels of haptoglobin and thrombocytes were observed after 28 weeks [6]. These inflammatory associations need to be further explored with larger study cohorts to distinguish if specific dietary components of the diet could be responsible for proand anti-inflammatory effects. Components suggested to be important as anti-inflammatory are unsaturated fatty acids and vitamins [24, 25], ingredients frequently used in the Mediterranean, O-BN, and healthy Nordic diets.

Previous studies examining the effect of diet on cognitive function have not examined the O-BN diet specifically, but the Mediterranean diet, which shares many features 
with the O-BN diet. Even though studies show inconsistent results, collective evidence suggests a protective role for the Mediterranean diet against dementia and cognitive decline. Causality has, however, not been established since most studies are observational [29]. No test of cognitive function was performed prior or after study start. The metabolic syndrome, diabetes and obesity are coupled to cognitive failure and $\mathrm{AD}$, where factors such as glucose and insulin levels, lipid parameters, blood pressure and body weight are supposed to be involved [30]. In the pathophysiology behind development of dementia in these lifestyle diseases, IL-18 is speculated to play a key role. IL-18 is supposed to enter the brain through a leaky blood brain barrier (BBB), which may depend on increased activity of metalloproteinases (MMP). Since IL-18 is a pro-inflammatory factor, it enhances the oxidative stress, neuritic amyloid- $\beta$ (A $\beta$ ) plaque formation and hyperphosphorylation of tau $[11,30]$. Thus, the lower levels of IL-18 observed after dietary intervention may be crucial for a reduced chronic inflammation and improved cognitive function. The improved HRQoL with greater physical functioning and vitality and psychological well-being may hypothetically also depend on lower IL-18 levels [7]. In the breakfast study, no significant differences in postprandial inflammation could be observed between the two groups receiving dissimilar breakfasts. Since diets with a higher glucose-content have been shown to induce postprandial inflammation [2], we hypothesized that the reference breakfast (which had a higher sugar content) might induce a more powerful inflammatory response. This was, however, not the case. Upon examination of cytokine concentrations after both meals, we could establish no clear pattern of response. Experimental trials in rats have shown that inhibition of postprandial hyperglycemia reduced the expression of genes coding for IL-18 in peripheral leukocytes [31]. Previous human studies have shown that acutely induced hyperglycemia increased levels of IL-18 in both healthy controls and patients with impaired glucose tolerance [32]. The decreased postprandial glucose and triglyceride levels after the single test breakfast, with comparable cytokine levels after both 
breakfasts, suggest that the improved metabolic control preceded lower levels of proinflammatory cytokines after long-term administration of the O-BN diet.

NfL concentrations increased significantly with the dietary intervention. A possible explanation for the NfL increase observed could pertain to the mental and physical stress exerted on study participants during the dietary intervention. Perhaps the resulting catabolic state from dietary intervention resulted in strain and subsequent damage on the brain, through an unknown mechanism. It should be noted however, that the increase was minor and within the concentration range observed in neurologically healthy individuals (ref 13 Mattsson et al. + PMID: 29070659). A previous 10-year long behavioral interventional study led to weight loss but showed marginally greater cognitive decline in T2DM study participants compared to controls [33]. The authors hypothesized that chronic hypoglycemia or decreased levels of the adipokine leptin could explain these results. Leptin has been proposed to have neuroprotective properties, such as promoting neurogenesis and attenuating apoptosis in the brain [34]. Interestingly enough, in the present study leptin was decreased after the 12-week dietary intervention, as previously reported [6]. Alternatively, the dietary intervention might have had effects on peripheral nerves that also contain NfL. Yet another possibility is that the dietary intervention might have induced myelin changes that in turn could translate into increased release of NfL. In any case, the result of increased NfL levels after dietary intervention should be regarded with caution and far-drawn conclusions should be avoided, not least since NfL, to our knowledge, has not been used previously as a biomarker of cognitive function in the context of a study like this. In the future, it would be interesting to combine cognitive testing with measurements of IL-18 and NfL to further elucidate biomarker accuracy as well as the effect of the O-BN diet on cognitive function. Knyta ihop IL-18 och neurofilament, Oskar, Henrik och Elisabet 
Cytokines are small peptide molecules involved in a variety of processes, including humoral and cell-mediated responses of the immune system through cell signaling, both locally as well as systemically [35]. Since cytokines were measured in blood, their local effects were not considered. There is a possibility that para- and autocrine effects are of more importance than systemic effect in the context of inflammatory status in metabolic disease. An alternative method used in several studies is to examine adipose tissue concentrations [36, 37].

Concentrations of soluble markers of inflammation display a wide interindividual variation, which is modified by a number of factors such as age, body fat, physical activity, sex and genetics, to name a few [8]. It should, nonetheless, be highlighted that in this dietary intervention study, the same set of individuals were followed over time, which excludes some of the mentioned characteristics as confounding factors. The mean age of the control group was higher than in the T2D group which, if anything, should have led to elevated levels of inflammatory biomarkers in the control group [2]. Because of the natural variation of cytokine levels and the fact that concentrations between healthy and obese individuals tend to overlap, it may be that larger study groups are required to obtain statistically significant results. The power of the present study was calculated for metabolic rather than inflammatory parameters.

Many samples had non-detectable concentrations due to the typically low concentrations present in body fluids, which likely further affected results. Cytokines have a high biological activity and, as a consequence of this, their homeostatic concentration is tightly regulated [35]. Remaining variables that could have influenced results include, most importantly, the weight loss that was observed in study participants. Since weight reduction per $s e$ reduces systemic inflammation [2], this naturally needs to be taken into consideration when evaluating effect and assessing causality of the diet on inflammatory parameters. Due to the 
small sample size, logistic regression could not be performed, and thus, the independent influence of the different variables on IL-18 could not be determined.

\section{Conclusion}

A single O-BN-based breakfast to healthy volunteers led to lower postprandial glucose and triglyceride levels compared with reference breakfast, without affecting the cytokine concentrations. A 12-week O-BN dietary intervention to 2TD rendered lower plasma levels of IL-18, in parallel with improved levels of fasting glucose, HbA1c, and lipid values. NfL levels in plasma increased during the intervention.

\section{Acknowledgements}

We want to acknowledge Igelösa Life Science AB, Lund, whose staff supplied and prepared the meals, and the staff at the Internal Medicine Research Group, Skåne University Hospital, Malmö, for performing the trials. We also want to acknowledge William Critchley, Timothy Entwistle and James Fildes, The Manchester Collaborative Centre for Inflammation Research, University Hospital of South Manchester, Manchester, UK, for assistance with analyses of cytokines.

\section{Funding}

This study was supported by grants from the Royal, Physiographic Society in Lund, HansGabriel and Alice Trolle Wachtmeister' s Foundation for Medical Research, King Gustaf V:s and queen Victoria Free Maison `s Foundation, Dir Albert Påhlsson ’s Foundation, Development Foundation of Region Skåne, Foundation of Skåne University Hospital, the Swedish and European Research Councils and the Knut and Alice Wallenberg Foundation.

\section{Author's contribution}

$\mathrm{BR}, \mathrm{PH}$, and $\mathrm{BO}$ planned and designed the study, and financed the analyses. $\mathrm{CN}$ and $\mathrm{BR}$ performed the cytokine analyses. $\mathrm{CN}$ performed the statistical analyses together with $\mathrm{PH}$ and 
wrote the initial draft together with BO. EE, OH and $\mathrm{HZ}$ planned and analyzed the NfL

analyses. All authors contributed to the intellectual analyses of the manuscript and approved the final version.

Conflicts of Interest: HZ is one of the founders of Brain Biomarker Solutions in Gothenburg $\mathrm{AB}$, a GU Ventures-based platform company at the University of Gothenburg, and has served at advisory boards of Roche Diagnostics and Eli Lilly. The other authors declare no conflict of interest.

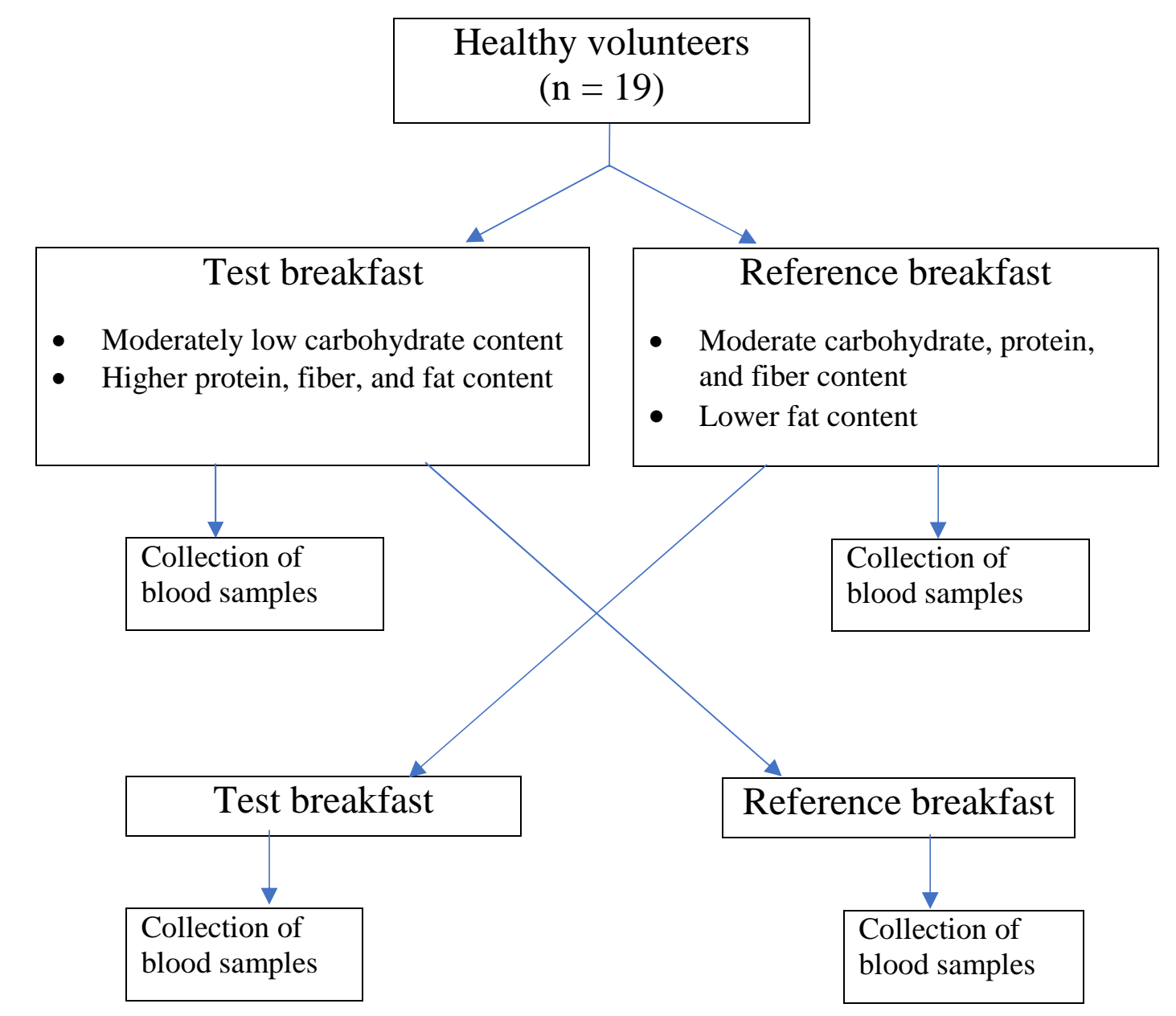


Figure 1. Individual flow chart for short interventional (breakfast) study

One participant was excluded due to missing samples. Participants ingested the different breakfasts on 2 different days: (1) the traditional reference breakfast (2) and the Okinawan-based Nordic test breakfast. The meals were served in a random order at 1-week intervals. Blood samples were collected through an intravenous catheter 10 minutes before the meal, immediately before the meal, and 10, 20, 30, 45, 60, 90, 120 , and 180 minutes after the meal.

Table 1. Characteristics of the two studies

\begin{tabular}{|l|l|l|}
\hline Study design & \multicolumn{1}{|c|}{ Breakfast study } & \multicolumn{1}{c|}{$\begin{array}{c}\text { Dietary interventional (O-BN } \\
\text { diet) study }\end{array}$} \\
\hline Study population & Healthy volunteers $(\mathrm{n}=19)$ & $\begin{array}{l}\text { T2D patients (n = 30 at 12 weeks } \\
\text { and } \mathrm{n}=23 \text { at 28 weeks). Healthy } \\
\text { controls at baseline (n = 25) }\end{array}$ \\
\hline $\begin{array}{l}\text { Type of dietary } \\
\text { intervention }\end{array}$ & $\begin{array}{l}\text { 2 different breakfasts (one O-BN- } \\
\text { based and one standard) ingested } \\
\text { at two separate time points }\end{array}$ & $\begin{array}{l}\text { 12 week long dietary intervention } \\
\text { with the O-BN diet }\end{array}$ \\
\hline $\begin{array}{l}\text { Total participant } \\
\text { study time }\end{array}$ & 7 hours (3.5 h per breakfast) & 28 weeks \\
\hline $\begin{array}{l}\text { Measured } \\
\text { parameters } \\
\text { (time-points for } \\
\text { measurement) }\end{array}$ & $\begin{array}{l}\text { Inflammatory biomarkers (pre- } \\
\text { and postprandial) }\end{array}$ & $\begin{array}{l}\text { Inflammatory biomarkers, NfL } \\
\text { (baseline, 12 weeks, 28 weeks) }\end{array}$ \\
\hline
\end{tabular}

O-BN diet $=$ Okinawan-based Nordic diet, T2D = type 2 diabetes . 
Table 2. Anthtropometric and metabolic characteristics of the type 2 cohort

\begin{tabular}{|l|c|c|}
\hline Variable & Mean \pm SD & P-value \\
\hline BMI $\left(\mathrm{kg} / \mathrm{m}^{2}\right)$ & & \\
\hline Baseline & $29.9 \pm 4.1$ & \\
\hline Week 12 & $27.8 \pm 3.9$ & $<0.001$ \\
\hline Week 28 & $28.4 \pm 4.6$ & $<0.001$ \\
\hline Fasting glucose $(\mathrm{mmol} / \mathrm{L})$ & & \\
\hline Baseline & $9.7 \pm 3.5$ & $<0.001$ \\
\hline Week 12 & $7.9 \pm 2.7$ & 0.47 \\
\hline Week 28 & $9.3 \pm 3.3$ & \\
\hline HbA1c $(\mathrm{mmol} / \mathrm{mol})$ & & $<0.001$ \\
\hline Baseline & $61.6 \pm 17.6$ & 0.002 \\
\hline Week 12 & $49.2 \pm 10.4$ & \\
\hline Week 28 & $54.4 \pm 13.3$ & $<0.001$ \\
\hline Insulin (mIU/L) & & 0.064 \\
\hline Baseline & $15.5 \pm 9.7$ & \\
\hline Week 12 & $11.7 \pm 6.7$ & \\
\hline Week 28 & $12.8 \pm 7.4$ & 0.04 \\
\hline Triglycerides (mmol/L) & & \\
\hline Baseline & $1.8 \pm 1.1$ & \\
\hline Week 12 & $1.5 \pm 0.9$ & \\
\hline Week 28 & $2.0 \pm 1.6$ & \\
\hline Cholesterol $(\mathrm{mmol} / \mathrm{L})$ & & \\
\hline Baseline & $4.7 \pm 0.8$ & \\
\hline Week 12 & $4.2 \pm 1.0$ & \\
\hline Week 28 & $4.7 \pm 0.8$ & \\
\hline
\end{tabular}

$\mathrm{N}=30$ subjects. The mean values and standard deviations (SD) are presented for body-mass index (BMI), fasting blood values of hemoglobin A1c (HbA1c); fasting serum levels of insulin; and fasting plasma values of glucose, triglycerides and cholesterol. Values are shown at inclusion (baseline), 12 weeks after diet intervention and 16 weeks after the end of diet intervention (week 28, $\mathrm{n}=23$ ). Comparisons were made between each time point and baseline, using linear mixed effect models. P-value $<0.05$ was considered statistically significant. 
Table 3. Pre- and postprandial cytokine concentrations in the breakfast study

\begin{tabular}{|c|c|c|c|c|c|c|}
\hline & Test breakfast & & Reference & & Test - Reference & \\
\hline Variable & Median (IQR) & $\mathrm{P}$-value & Median (IQR) & P-value & Median of difference & $\mathrm{P}$-value \\
\hline \multicolumn{7}{|l|}{ TNF- $\alpha$} \\
\hline baseline & $29.8(0.65-53.4)$ & & $32.5(3.0-3.6)$ & & $0(-2.3-1.8)$ & 0.78 \\
\hline $\operatorname{Max}$ & $30.5(2.3-63.7)$ & 0.053 & $34.0(0.01-56.8)$ & 0.22 & $0(-0.8-2.3)$ & 0.30 \\
\hline Min & $28.5(0.2-56.3)$ & 0.006 & $29.4(0.01-48.7)$ & 0.003 & $0(-0.2-0.1)$ & 0.26 \\
\hline$d M a x$ & $1.0(-0.6-3.9)$ & & $0(0-2.5)$ & & $0(-1.8-4.0)$ & 0.46 \\
\hline$d M i n$ & $1.9(0-4.5)$ & & $1.4(0-5.4)$ & & $0(-2.3-1.4)$ & 0.59 \\
\hline \multicolumn{7}{|l|}{ IL-1 $\beta$} \\
\hline baseline & $15.0(0.01-40.3)$ & & $14.3(0.01-41.4)$ & & $0(0-1.8)$ & 0.51 \\
\hline $\operatorname{Max}$ & $21.4(0.01-43.8)$ & 0.12 & $13.8(0.01-44.7)$ & 0.07 & $0(-1.1-1.2)$ & 0.73 \\
\hline Min & $11.4(0.01-29.6)$ & 0.013 & $11.7(0.01-34.6)$ & 0.009 & $0(-0.4-1.1)$ & 0.40 \\
\hline$d M a x$ & $0(0-3.5)$ & & $0.8(0-1.8)$ & & $0(-1.5-3.1)$ & 0.78 \\
\hline$d M i n$ & $0.9(0-4.2)$ & & $0.7(0-3.5)$ & & $0(0-2.1)$ & 0.65 \\
\hline \multicolumn{7}{|l|}{ IFN-Y } \\
\hline baseline & $667(1.2-1230)$ & & $611(8.8-1430)$ & & $0(-84.4-68.0)$ & 0.69 \\
\hline $\operatorname{Max}$ & $957(59.2-1370)$ & 0.013 & $691(7.0-1480)$ & 1.0 & $0(-38.3-41.2)$ & 1.0 \\
\hline Min & $620(51.0-1282)$ & 0.04 & $585(2.5-1271)$ & 0.02 & $0(-10.7-33.9)$ & 0.88 \\
\hline$d M a x$ & $18.2(0-75.5)$ & & $0(-43.0-66.1)$ & & $11.1(-51.6-121.3)$ & 0.26 \\
\hline$d M i n$ & $14.0(0-115)$ & & $13.6(0-94.2)$ & & $0(-80.2-115.1)$ & 1.0 \\
\hline \multicolumn{7}{|l|}{ IL-2 } \\
\hline baseline & $392(0.01-1080)$ & & $454(0.01-860)$ & & $0(-21.4-78.4)$ & 0.59 \\
\hline $\operatorname{Max}$ & $438(0.01-1230)$ & 0.64 & $431(0.01-1050)$ & 0.20 & $0(-17.8-8.2)$ & 0.68 \\
\hline Min & $335(0.01-1023)$ & 0.01 & $332(0.01-994)$ & 0.08 & $0(-10.1-27.5)$ & 0.83 \\
\hline$d \operatorname{Max}$ & $0(-6.6-10.2)$ & & $3.4(0-53.4)$ & & $0(-72.1-60.6)$ & 0.88 \\
\hline$d M i n$ & $17.1(0-78.0)$ & & $20.3(0-62.2)$ & & $0(-14.8-64.1)$ & 0.68 \\
\hline \multicolumn{7}{|l|}{ IL-12p70 } \\
\hline baseline & $544(0.01-1030)$ & & $550(0.01-1050)$ & & $0(-0.2-38.3)$ & 0.83 \\
\hline $\operatorname{Max}$ & $550(0.01-1160)$ & 0.16 & $543(0.01-1080)$ & 0.25 & $-8.8(-33.0-0)$ & 0.40 \\
\hline Min & $493(0.01-994)$ & 0.002 & $518(0.01-915)$ & 0.004 & $0(-24.4-28.5)$ & 0.47 \\
\hline$d M a x$ & $1.5(0-35.1)$ & & $1.8(0-20.7)$ & & $0(-37.1-34.5)$ & 0.73 \\
\hline dMin & $19.6(0-50.6)$ & & $26.3(0-92.6)$ & & $0(-51.2-18.4)$ & 0.59 \\
\hline \multicolumn{7}{|l|}{ IL-18 } \\
\hline baseline & $219(168-309)$ & & $215(170-311)$ & & $4.9(-26.7-15.4)$ & 0.97 \\
\hline Max & $222(178-333)$ & 0.36 & $208(175-294)$ & 1.0 & $-4.6(-21.8-17.8)$ & 0.87 \\
\hline Min & $197(168-264)$ & 0.003 & $188(160-281)$ & $<0.001$ & $2.0(-11.4-17.7)$ & 0.72 \\
\hline$d M a x$ & $1.9(-7.7-21.7)$ & & $-0.5(-9.1-13.1)$ & & $4.5(-24.8-50.4)$ & 0.38 \\
\hline$d M i n$ & $12.8(2.5-30.5)$ & & $21.0(9.4-35.7)$ & & $-5.2(-28.8-17.7)$ & 0.49 \\
\hline \multicolumn{7}{|l|}{ IL-4 } \\
\hline baseline & $108(0.01-170)$ & & $108(0.01-157)$ & & $0(-3.3-8.0)$ & 0.51 \\
\hline $\operatorname{Max}$ & $113(0.01-204)$ & 0.17 & $105(0.01-162)$ & 0.20 & $0(-5.2-0)$ & 0.30 \\
\hline Min & $102(0.01-152)$ & 0.002 & $95.8(0.01-146)$ & 0.034 & $0(-11.4-17.7)$ & 0.28 \\
\hline$d M a x$ & $0(-0.5-4.2)$ & & $0(-0.5-8.6)$ & & $0(-8.6-0.1)$ & 0.55 \\
\hline$d M i n$ & $3.7(0-8.7)$ & & $5.1(0-11.9)$ & & $0(-5.1-8.1)$ & 0.64 \\
\hline
\end{tabular}

$\mathrm{n}=19$. Plasma concentrations of cytokines $(\mathrm{pg} / \mathrm{mL})$ were measured after ingestion of the Okinawan-based Nordic test breakfast and the traditional reference breakfast. Values are presented as the median value and interquartile range (IQR) for baseline, postprandial minimal and maximal levels and for minimal and maximal changes from baseline, delta min (dmin), and delta max (dmax). Median values and IQR are provided for differences between the two meals. Wilcoxon test was used for comparison between baseline and maximal and minimal levels, and between meals. P-value $<0.05$ was considered statistically significant. 
Table 4. Inflammatory parameters and NfL concentrations in T2D patients and nondiabetic controls

\begin{tabular}{|c|c|c|c|}
\hline Variable & $\begin{array}{c}\text { T2D patients } \\
\mathbf{N}=\mathbf{3 0}\end{array}$ & $\begin{array}{c}\text { Non-diabetic } \\
\text { controls } \\
\mathbf{N}=\mathbf{2 5} \\
\end{array}$ & P-value \\
\hline Age (years) & $57.5 \pm 8.2$ & $64.7 \pm 5.3$ & 0.001 \\
\hline Body mass index $\left(\mathrm{kg} / \mathrm{m}^{2}\right)$ & $29.9 \pm 4.1$ & $24.1 \pm 3.6$ & $<0.001$ \\
\hline \multicolumn{4}{|c|}{ P-Cytokines and NFL $(\mathrm{pg} / \mathrm{mL})$} \\
\hline TNF- $\alpha$ & $5.2(1.5-18.6)$ & $1.2(0.03-6.4)$ & $<0.001$ \\
\hline IFN- $\gamma$ & $82.8(11.1-376)$ & $45.7(3.6-261)$ & 0.018 \\
\hline IL-2 & $7.5(4.9-436)$ & $59.4(27.1-231)$ & $<0.001$ \\
\hline IL-12p70 & $237(20.8-621)$ & $38.8(3.3-319)$ & $<0.001$ \\
\hline IL-18 & $167(112-277)$ & $92.7(65.9-178)$ & 0.30 \\
\hline IL-4 & $24.3(2.4-161)$ & $5.2(3.0-56.0)$ & $<0.001$ \\
\hline NFL & $16.1(10.6-26.4)$ & $21.0(16.8-32.4)$ & 0.075 \\
\hline \multicolumn{4}{|c|}{ Other inflammatory markers } \\
\hline $\begin{array}{l}\text { B-Hemoglobin }(\mathrm{g} / \mathrm{L}) \\
\text { Missing values: } 1^{*} .1^{* *}\end{array}$ & $141(134-152)$ & $131(126-137)$ & 0.001 \\
\hline $\begin{array}{l}\text { B-Leukocytes }\left(x 10^{9} / \mathrm{L}\right) \\
\text { Missing values: } 1^{*}, 4^{* *}\end{array}$ & $6.3(5.5-7.4)$ & $6.8(5.3-7.8)$ & 0.94 \\
\hline $\begin{array}{l}\text { B-Thrombocytes }\left(\times 10^{9} / \mathrm{L}\right) \\
\text { Missing values: } 1^{*}, 7^{* *}\end{array}$ & $255(221-302)$ & $248(201-293)$ & 0.63 \\
\hline P-Albumin (g/L) & $39.0(37.0-41.3)$ & $40.0(38.5-43.5)$ & 0.13 \\
\hline P-CRP (mg/L) & $2.2(1.0-3.2)$ & $2.0(1.0-3.5)$ & 0.89 \\
\hline
\end{tabular}

Plasma (P) and blood (B) levels of cytokines, neurofilament light (NfL), hemoglobin, leukocytes, thrombocytes, albumin and C-reactive protein (CRP) concentrations in healthy controls and type 2 diabetes (T2D) patients at baseline. For parameters with missing values; these are presented for *the T2D patient group, and **the non-diabetic control group under each parameter. Mean \pm standard deviation (SD) or median values and interquartile ranges (IQR) are presented. Student t-test or Mann-Whitney U-test. P-value <0.05 was considered statistically significant. 
Table 5. Cytokine and NfL concentrations in dietary interventional study

\begin{tabular}{|c|c|c|c|c|c|c|c|}
\hline Variable & Mean & LCL & $\mathbf{U C L}$ & $\begin{array}{l}\text { Mean } \\
\text { difference }\end{array}$ & LCL & UCL & $P$-value \\
\hline \multicolumn{8}{|l|}{ TNFa } \\
\hline Baseline & 14.9 & 6.2 & 23.6 & & & & \\
\hline 12 weeks & 12.5 & 3.8 & 21.2 & -2.4 & -5.6 & 0.8 & 0.14 \\
\hline 28 weeks & 13.6 & 4.8 & 22.5 & -1.3 & -6.0 & 3.5 & 0.60 \\
\hline \multicolumn{8}{|l|}{ IL1 $\beta$} \\
\hline Baseline & 12.1 & 4.6 & 19.6 & & & & \\
\hline 12 weeks & 10.3 & 3.7 & 31.1 & -1.7 & -4.3 & 0.8 & 0.17 \\
\hline 28 weeks & 10.8 & 3.7 & 33.2 & -1.3 & -5.1 & 2.5 & 0.49 \\
\hline \multicolumn{8}{|l|}{ IFN $\gamma$} \\
\hline Baseline & 778 & -143 & 1699 & & & & \\
\hline 12 weeks & 643 & -278 & 1564 & -135 & -307 & 37.3 & 0.12 \\
\hline 28 weeks & 656 & -269 & 1581 & -122 & -382 & 138 & 0.35 \\
\hline \multicolumn{8}{|l|}{ IL-2 } \\
\hline Baseline & 315 & 89.7 & 541 & & & & \\
\hline 12 weeks & 321 & 95.6 & 547 & 5.9 & -60.5 & 72.2 & 0.86 \\
\hline 28 weeks & 357 & 129 & 585 & 41.5 & -58.0 & 141 & 0.41 \\
\hline \multicolumn{8}{|l|}{ IL-12p70 } \\
\hline Baseline & 490 & 223 & 756 & & & & \\
\hline 12 weeks & 415 & 148 & 682 & -74.6 & -157 & 7.3 & 0.07 \\
\hline 28 weeks & 458 & 188 & 728 & -31.3 & -154 & 91.4 & 0.61 \\
\hline \multicolumn{8}{|l|}{ IL-18 } \\
\hline Baseline & 226 & 173 & 280 & & & & \\
\hline 12 weeks & 189 & 136 & 242 & -37.7 & -57.4 & -17.9 & 0.001 \\
\hline 28 weeks & 201 & 147 & 255 & -25.3 & -54.7 & 4.1 & 0.09 \\
\hline \multicolumn{8}{|l|}{ IL-4 } \\
\hline Baseline & 98.1 & 48.6 & 148 & & & & \\
\hline 12 weeks & 79.9 & 30.3 & 129 & -18.3 & -44.3 & 7.8 & 0.17 \\
\hline 28 weeks & 89.6 & 38.4 & 141 & -8.5 & -46.5 & 29.5 & 0.66 \\
\hline \multicolumn{8}{|l|}{ IL-1 $\alpha$} \\
\hline Baseline & 6.6 & 2.1 & 11.1 & & & & \\
\hline 12 weeks & 5.6 & 1.2 & 10.1 & -1.0 & -2.4 & 0.5 & 0.18 \\
\hline 28 weeks & 6.0 & 1.4 & 10.5 & -0.6 & -2.8 & 1.5 & 0.56 \\
\hline \multicolumn{8}{|l|}{ NFL } \\
\hline Baseline & 21.0 & 15.3 & 26.7 & & & & \\
\hline 12 weeks & 26.2 & 18.5 & 33.8 & 5.2 & 0.02 & 10.3 & 0.049 \\
\hline 28 weeks & 23.4 & 16.7 & 30.2 & 2.4 & -1.1 & 6.0 & 0.18 \\
\hline
\end{tabular}

$\mathrm{n}=30$ at week 12 and $\mathrm{n}=23$ at week 28. Cytokine and neurofilament light (NfL) concentrations $(\mathrm{pg} / \mathrm{mL})$ in blood plasma were measured at baseline as well as 12 and 28 weeks after dietary intervention. Mean values, lower (LCL) and upper (UCL) 95\% confidence levels are provided for each time point. Mean values, LCL and UCL (95\% confidence level) are provided for changes from baseline to 12 and 28 weeks. Mixed effect models. P-value $<0.05$ was considered statistically significant. 
Table 6. Correlations between interleukin-18 and anthropometric and metabolic parameters

\begin{tabular}{|c|c|c|c|}
\hline & \multicolumn{3}{|c|}{ Interleukin-18 } \\
\hline Variables & Baseline & 12 weeks & 28 weeks \\
\hline Body mass index & $\mathrm{rs}=0.209, \mathrm{p}=0.268$ & $\mathrm{rs}=0.198, \mathrm{p}=0.313$ & $\mathrm{rs}=0.276, \mathrm{p}=0.203$ \\
\hline P-Glucose & $r s=0.446, p=0.014$ & $\mathrm{rs}=0.362, p=0.049$ & $r s=0.578, p=0.004$ \\
\hline B-HbA1c & $\mathrm{rs}=0.411, p=0.024$ & $\mathrm{rs}=0.424, p=0.019$ & $\mathrm{rs}=0.412, \mathrm{p}=\mathbf{0 . 0 5 1}$ \\
\hline S-Insulin & $\mathrm{rs}=-0.240, \mathrm{p}=0.202$ & $\mathrm{rs}=0.015, \mathrm{p}=0.937$ & $\mathrm{rs}=-0.090, \mathrm{p}=0.689$ \\
\hline P-Cholesterol & $\mathrm{rs}=-0.194, \mathrm{p}=0.305$ & $\mathrm{rs}=0.617, \mathrm{p}<0.001$ & rs $=0.751, p<0.001$ \\
\hline P-Triglycerides & $\mathrm{rs}=0.525, \mathrm{p}=0.003$ & $\mathrm{rs}=0.554, \mathrm{p}=0.001$ & $r s=0.478, p=0.021$ \\
\hline $\begin{array}{l}\text { Systolic blood } \\
\text { pressure }\end{array}$ & $\mathrm{rs}=0.101, \mathrm{p}=0.596$ & $\mathrm{rs}=0.262, \mathrm{p}=0.178$ & $\mathrm{rs}=0.347, \mathrm{p}=0.105$ \\
\hline $\begin{array}{l}\text { Diastolic blood } \\
\text { pressure }\end{array}$ & $\mathrm{rs}=0.016, \mathrm{p}=0.934$ & $\mathrm{rs}=0.179, \mathrm{p}=0.362$ & $\mathrm{rs}=0.265, \mathrm{p}=0.222$ \\
\hline
\end{tabular}

HbA1c $=$ hemoglobin A1c $($ HbA1c) in blood, $\mathrm{P}=$ plasma, $\mathrm{S}=$ serum. Spearman's correlation test. $\mathrm{P}<005$ was considered statistically significant. 


\section{References}

1.

860-867.

2.

Hotamisligil, G.S. Inflammation and metabolic disorders. Nature 2006, 444,

K.; Esposito, K.; Jönsson, L.S.; Kolb, H.; Lansink, M.; Marcos, A.; Margioris, A.;

Matusheski, N.; Nordmann, H.; O'Brien, J.; Pugliese, G.; Rizkalla, S.; Schalkwijk, C.;

Tuomilehto, J.; Wärnberg, J.; Watzl, B.; Winklhofer-Roob, B.M. Dietary factors and lowgrade inflammation in relation to overweight and obesity. Br J Nutr 2011,106 Suppl 3, S5-78.

3. Uusitupa, M.; Schwab, U. Diet, inflammation and prediabetes-impact of quality of diet. Can J Diabetes 2013, 37, 327-331.

4. Ohlsson, B.; Hoglund, P.; Roth, B.; Darwiche, G. Modification of a traditional breakfast leads to increased satiety along with attenuated plasma increments of glucose, Cpeptide, insulin, and glucose-dependent insulinotropic polypeptide in humans. Nutr Res 2016, 36, 359-368.

5. Ohlsson, B.; Darwiche, G.; Roth, B.; Hoglund, P. Two meals with different carbohydrate, fat and protein contents render equivalent postprandial plasma levels of calprotectin, cortisol, triglycerides and zonulin. Int J Food Sci Nutr 2016, 67, 872-880.

6. Ohlsson, B.; Roth, B.; Larsson, E.; Hoglund, P. Calprotectin in serum and zonulin in serum and feces are elevated after introduction of a diet with lower carbohydrate content and higher fiber, fat and protein contents. Biomed Rep 2017, 6, 411-422.

$7 . \quad$ Darwiche, G.; Hoglund, P.; Roth, B.; Larsson, E.; Sjoberg, T.; Wohlfart, B.; Steen, S.; Ohlsson, B. An Okinawan-based Nordic diet improves anthropometry, metabolic control, and health-related quality of life in Scandinavian patients with type 2 diabetes: a pilot trial. Food Nutr Res 2016, 60, 32594.

8. $\quad$ Calder, P.C.; Ahluwalia, N.; Albers, R.; Bosco, N.; Bourdet-Sicard, R.; Haller D,; Holgate, S.T.; Jönsson, L.S.; Latulippe, M.E.; Marcos, A.; Moreines, J.; M'Rini, C.;

Müller, M.; Pawelec, G.; van Neerven, R.J.; Watzl, B.; Zhao, J. A consideration of biomarkers to be used for evaluation of inflammation in human nutritional studies. Brit $\mathrm{J}$ Nutr 2013, 109 Suppl 1, S1-34.

9. Ojo, O.; Brooke, J. Evaluating the Association between Diabetes, Cognitive Decline and Dementia. Int J Environ Res Public Health 2015, 12, 8281-8294.

10. Ninomiya, T. Diabetes mellitus and dementia. Curr Diabet Rep 2014, 14, 487.

11. Bharadwaj, P.; Wijesekara, N.; Liyanapathirana, M.; Newsholme, P.; Ittner, L.; Fraser, P.; Verdile, G. The Link between Type 2 Diabetes and Neurodegeneration: Roles for Amyloid-beta, Amylin, and Tau Proteins. J Alzheimer's Dis 2017, 59, 421-432.

12. Steen, E.; Terry, B.M.; Rivera, E.J.; Cannon, J.L.; Neely, T.R.; Tavares, R.; Xu, X.J.; Wands, J.R.; de la Monte, S.M. Impaired insulin and insulin-like growth factor expression and signaling mechanisms in Alzheimer's disease--is this type 3 diabetes? $J$ Alzheimer's Dis 2005, 7, 63-80.

13. Mattsson, N.; Andreasson, U.; Zetterberg, H.; Blennow, K. Alzheimer's Disease Neuroimaging I. Association of plasma neurofilament light with neurodegeneration in patients with alzheimer disease. JAMA Neurology 2017, 74, 557-566.

14. Nordic Council of Ministers. Nordic Nutrition Recommendations 2012 [cited 2017-11-01]. Available from: http://www.norden-ilibrary.org/social-issues-migrationhealth/nordic-nutrition-recommendations-2012_nord2014-002.

15. Bantle, J.P.; Wylie-Rosett, J.; Albright, A.L.; Apovian, C.M.; Clark, N.G.; Franz, M.J.; Hoogwerf, B.J.; Lichtenstein, A.H.; Mayer-Davis, E.; Mooradian, A.D.; Wheeler, 
M,L. Nutrition recommendations and interventions for diabetes: a position statement of the American Diabetes Association. Diabetes Care 2008, 31 Suppl 1, S61-78.

16. Ryden, L.; Grant, P.J.; Anker, S.D.; Berne, C.; Cosentino, F.; Danchin, N.; Deaton, C.; Escaned, J.; Hammes, H.P.; Huikuri, H.; Marre, M.; Marx, N.; Mellbin, L.; Ostergren, J.; Patrono, C.; Seferovic, P.; Uva, M.S.; Taskinen, M.R.; Tendera, M.;

Tuomilehto, J.; Valensi, P.; Zamorano, J.L. ESC guidelines on diabetes, pre-diabetes, and cardiovascular diseases developed in collaboration with the EASD - summary. Diab Vasc Dis Res 2014, 11, 133-173.

17. Galic, S.; Oakhill, J.S.; Steinberg, G.R. Adipose tissue as an endocrine organ. Mol Cell Endocrinol 2010, 316, 129-139.

18. Minihane, A.M.; Vinoy, S.; Russell, W.R.; Baka, A.; Roche, H.M.; Tuohy, K.M.; Teeling, J.L.; Blaak, E.E.; Fenech, M.; Vauzour, D.; McArdle, H.J.; Kremer, B.H.; Sterkman, L.; Vafeiadou, K.; Benedetti, M.M.; Williams, C.M.; Calder, P.C. Low-grade inflammation, diet composition and health: current research evidence and its translation. Brit J Nutr 2015, 114, 999-1012.

19. Banchereau, J.; Pascual, V.; O'Garra, A. From IL-2 to IL-37: the expanding spectrum of anti-inflammatory cytokines. Nature immunology 2012, 13, 925-931.

20. Gaffen, S.L.; Liu, K.D. Overview of interleukin-2 function, production and clinical applications. Cytokine 2004, 28, 109-123.

21. Stensvold, D.; Slordahl, S.A.; Wisloff, U. Effect of exercise training on inflammation status among people with metabolic syndrome. Metab Syndr Relat Disord 2012, 10, 267-272.

22. Esposito, K.; Pontillo, A.; Di Palo, C.; Giugliano, G.; Masella, M.; Marfella, R.; Giugliano, D. Effect of weight loss and lifestyle changes on vascular inflammatory markers in obese women: a randomized trial. JAMA 2003, 289, 1799-1804.

23. Bruun, J.M.; Helge, J.W.; Richelsen, B.; Stallknecht, B. Diet and exercise reduce low-grade inflammation and macrophage infiltration in adipose tissue but not in skeletal muscle in severely obese subjects. Am J Physiol Endocr Metab 2006, 290, E961-967. 24. Troseid, M.; Arnesen, H.; Hjerkinn, E.M.; Seljeflot, I. Serum levels of interleukin-18 are reduced by diet and n-3 fatty acid intervention in elderly high-risk men. Metabolism 2009, 58, 1543-1549.

$25 . \quad$ Leder, L.; Kolehmainen, M.; Narverud, I.; Dahlman, I.; Myhrstad, M.C.; de Mello, V.D.; Paananen, J.; Carlberg, C.; Schwab, U.; Herzig, K.H.; Cloetens, L.; Storm, M.U.; Hukkanen, J.; Savolainen, M.J.; Rosqvist, F.; Hermansen, K.; Dragsted, L.O.; Gunnarsdottir, I.; Thorsdottir, I.; Risérus, U.; Åkesson, B.; Thoresen, M.; Arner, P.; Poutanen, K.S.; Uusitupa, M.; Holven, K.B.; Ulven, S.M. Effects of a healthy Nordic diet on gene expression changes in peripheral blood mononuclear cells in response to an oral glucose tolerance test in subjects with metabolic syndrome: a SYSDIET sub-study. Genes Nutr 2016, 11,3 .

26. Weiss, T.W.; Arnesen, H.; Troseid, M.; Kaun, C.; Hjerkinn, E.M.; Huber, K.; Wojta, J.; Seljeflot, I. Adipose tissue expression of interleukin-18 mRNA is elevated in subjects with metabolic syndrome and independently associated with fasting glucose. Wien Klin Wochenschr 2011, 123, 650-654.

27. Hivert, M.F.; Sun, Q.; Shrader, P.; Mantzoros, C.S.; Meigs, J.B.; Hu, F.B. Circulating IL-18 and the risk of type 2 diabetes in women. Diabetologia 2009, 52, 21012108.

28. Thorand, B.; Kolb, H.; Baumert, J.; Koenig, W.; Chambless, L.; Meisinger, C.; Illig, T.; Martin, S.; Herder, C. Elevated levels of interleukin-18 predict the development of type 2 diabetes: results from the MONICA/KORA Augsburg Study, 1984-2002. Diabetes 2005, 54, 2932-2938. 
29. Petersson, S.D.; Philippou, E. Mediterranean Diet, Cognitive Function, and Dementia: A Systematic Review of the Evidence. Adv Nutr 2016, 7, 889-904.

30. Ojala, J.O.; Sutinen, E.M. The Role of Interleukin-18, Oxidative Stress and Metabolic Syndrome in Alzheimer's Disease. J Clin Med 2017, 6, 55.

31. Imai, C.; Harazaki, T.; Inoue, S.; Mochizuki, K.; Goda, T. Inhibition of postprandial hyperglycemia by either an insulin-dependent or -independent drug reduces the expression of genes related to inflammation in peripheral leukocytes of OLETF rats. Biosci Biotech Biochem 2013, 77, 2305-2308.

32. $\quad$ Esposito, K.; Nappo, F.; Marfella, R.; Giugliano, G.; Giugliano, F.; Ciotola, M.; Quagliaro, L.; Ceriello, A.; Giugliano, D. Inflammatory cytokine concentrations are acutely increased by hyperglycemia in humans: role of oxidative stress. Circulation 2002, 106, 20672072.

33. $\quad$ Espeland, M.A.; Carmichael, O.; Hayden, K.; Neiberg, R.H.; Newman, A.B.; Keller, J.N.; Wadden, T.A.; Rapp, S.R.; Hill, J.O.; Horton, E.S.; Johnson, K.C.;

Wagenknecht, L.; Wing, R.R. Long-term Impact of Weight Loss Intervention on Changes in Cognitive Function: Exploratory Analyses from the Action for Health in Diabetes

Randomized Controlled Clinical Trial. J Gerontol A Biol Sci Med Sci 2017 Aug 25. doi: 10.1093/gerona/glx 165. [Epub ahead of print].

34. Kiliaan, A.J.; Arnoldussen, I.A.; Gustafson, D.R. Adipokines: a link between obesity and dementia? Lancet Neurology 2014, 13, 913-923.

35. Stenken, J.A.; Poschenrieder, A.J. Bioanalytical chemistry of cytokines--a review. Anal Chim Acta 2015, 853, 95-115.

36. $\quad$ You, T.; Wang, X.; Murphy, K.M.; Lyles, M.F.; Demons, J.L.; Yang, R.; Gong, D.W.; Nicklas, B.J. Regional adipose tissue hormone/cytokine production before and after weight loss in abdominally obese women. Obesity 2014, 22, 1679-1684.

37. Gruzdeva, O.V.; Akbasheva, O.E.; Dyleva, Y.A.; Antonova, L.V.; Matveeva, V.G.; Uchasova, E.G.; Fanaskova, E.V.; Karetnikova, V.N.; Ivanov, S.V.; Barbarash, O.L. Adipokine and Cytokine Profiles of Epicardial and Subcutaneous Adipose Tissue in Patients with Coronary Heart Disease. Bull Exp Biol Med 2017, 163, 608-611. 